\title{
Levantamento etnobotânico das plantas medicinais utilizadas pela comunidade de Inhamã, Pernambuco, Nordeste do Brasil
}

RODRIGUES, A.P. 1; ANDRADE, L.H.C. 1(")

1Universidade Federal de Pernambuco, Centro de Ciências Biológicas, Departamento de Botânica, Laboratório de Etnobotânica e Botânica Aplicada. Av. Prof. Moraes Rego s/n. CEP 50670-901. Cidade Universitária, Recife, PE, Brasil. laise@pq.cnpq.b

RESUMO: Avaliou-se o conhecimento, uso, obtenção e indicação terapêutica de plantas utilizadas pela comunidade rural de Inhamã, situada no município de Abreu e Lima, PE, Brasil. Foram realizadas entrevistas semi-estruturadas com 75 moradores. Foram citadas 155 espécies, distribuídas em 112 gêneros e 59 famílias. Conforme a indicação terapêutica, as espécies foram agrupadas em sistemas corporais reconhecidos pela Organização Mundial de Saúde e o índice de importância relativa (IR) foi calculado para cada espécie. Destacaram-se pelo número de espécies, as famílias: Asteraceae, Fabaceae e Lamiaceae. As doenças mais citadas estiveram relacionadas aos sistemas respiratório e gastrointestinal. Ocimum gratissimum, alfavaca, apresentou o maior valor de IR $(2,0)$, seguido por Lippia alba, erva cidreira $(1,6)$, Mentha villosa, hortelã pequena $(1,6)$ e Musa paradisiaca, banana $(1,5)$, indicadas para tratamento de transtornos dos sistemas respiratório, digestório e nervoso. Apenas $11 \%$ das espécies citadas tiveram prescrições médicas. Inhamã diferencia-se de outras comunidades próximas a centros urbanos porque os moradores cultivam a maioria das espécies medicinais que consomem.

Palavras-chave: Etnobotânica, flora medicinal, conhecimento tradicional, comunidades rurais

\begin{abstract}
An ethnobotanical survey of medicinal plants used by the rural community of Inhamã, state of Pernambuco, Northeastern Brazil. This study evaluated the extent of knowledge and use and the acquisition of medicinal plants in the rural community of Inhamã, located in the municipality of Abreu e Lima in the state of Pernambuco, Brazil. Medical indications for medicinal plants in this community were also characterized. We conducted semi-structured interviews with 75 community residents. A total of 155 plant species were reported, distributed among 112 genera and 59 families. Species were grouped by body system benefitted according to the code established by the World Health Organization, and relative importance (RI) was calculated for each species. The most represented families were Asteraceae, Fabaceae, and Lamiaceae. The majority of the disease symptoms reported was related to the respiratory and gastrointestinal systems. The Ocimum gratissimum, clove basil, presented the greatest RI value (2.0), followed by Lippia alba, bushy lippia (1.6), Mentha villosa, mint (1.6), and Musa paradisiaca, banana (1.5), all of which are used to treat ailments of the respiratory, gastrointestinal and nervous systems. Of the species reported, only $11 \%$ have medical indications. Inhamã differs from other communities located near urban centers, as the local residents cultivate the majority of the medicinal plants they use.
\end{abstract}

Keywords: ethnobotany, medicinal flora, traditional knowledge, rural peoples

\section{INTRODUÇÃO}

Nas pesquisas com enfoque etnobotânico o uso medicinal costuma ser a principal categoria quando se trata do estudo de populações situadas nos centros urbanos ou em comunidades rurais próximas a esses centros (Silva \& Andrade, 2005). Pesquisas nesta linha têm fornecido importante contribuição para o conhecimento da flora medicinal brasileira, desde a Região Norte até a Região Sul do país, como as realizadas por Garlet \& Irgang (2001), Fuck et al. (2005), Pilla et al. (2006), Marinho et al. (2011) e Zucchi et al. (2013). Analisandose em conjunto artigos elaborados para as cinco 
regiões do país percebe-se a constância de um conjunto de plantas, sejam as de origem europeia, como Rosmarinus officinalis L., ou africanas, como Ocimum gratissimum L., sejam as nativas do Brasil, como Anacardium occidentale L. (Silva \& Andrade, 2005). Todavia, observa-se que nos mesmos conjuntos a importância relativa das espécies varia, conforme as principais doenças que afligem a população local (Negrelle \& Fornazzari, 2007).

Os autores que pesquisam o uso popular de plantas medicinais frequentemente apontam a forte relação com a baixa renda dos usuários, assim como a concentração do conhecimento na faixa etária acima dos 50 anos e o fato de que este conhecimento não está mais sendo repassado (Puri \& Nair, 2004). Por esse motivo, é comum, mesmo entre moradores de comunidades rurais, que as plantas medicinais sejam adquiridas comercialmente ou por doação de poucos membros da comunidade que ainda preservam o hábito de cultivá-las (Pilla et al., 2006).

A eficácia comprovada da ação de várias plantas medicinais, como Schinus terebinthifolius Raddi (aroeira), Cymbopogon citratus (DC.) Stapf (capim santo), Plectranthus barbatus Andrews (boldo do mato), e o baixo custo do tratamento, têm despertado a atenção de alguns órgãos do Governo Federal, Estadual e Municipal, originando iniciativas públicas do tipo da desenvolvida junto a 12 municípios localizados no estado de São Paulo (Oliveira et al., 2006; Carvalho et al., 2013). Pesquisar quais as espécies medicinais conhecidas e utilizadas por uma determinada população urbana, periurbana ou rural, suas preferências, as formas de obtenção e preparo dos medicamentos, com certeza contribuirá para o sucesso de tais iniciativas, servindo de base para a seleção das plantas a serem indicadas por médicos nos postos de saúde locais.

Contribuindo para o conhecimento sobre o uso de plantas medicinais no Nordeste do Brasil, desenvolveu-se uma pesquisa junto à comunidade Inhamã, no município de Abreu e Lima, situado na Zona da Mata ao norte de Pernambuco. Após o levantamento das espécies, suas formas de uso e indicações terapêuticas, foram investigadas quais as de maior importância para a comunidade, suas formas de obtenção e práticas de manejo. Os dados obtidos poderão apoiar iniciativas do governo Municipal e Estadual para implantar e disseminar o uso desse recurso, oferecendo melhores condições de saúde à população local.

\section{METODOLOGIA}

Os primeiros povoados que deram origem ao município de Abreu e Lima (07 $54^{\circ} 42^{\prime \prime S}$ e $34 " 54$ '10"W, $19 \mathrm{~m}$ alt.) tiveram início no ano de 1540 e a formação definitiva se deu em 1838. O clima da região é quente e úmido e a vegetação original, do tipo Floresta subperenifólia, encontra-se reduzida a fragmentos de menos de 20 ha, intercalados por roçados e áreas ocupadas por edificações. Considerada uma das maiores cidades da Região Metropolitana do Recife, Abreu e Lima possui um comércio variado e um parque industrial crescente. O sistema de ensino e o comércio local são insuficientes para atender a todos, mas existe um bom sistema de transporte coletivo, proporcionando a oportunidade de exercerem tais atividades na capital. Assim, o contato de boa parte das crianças, jovens e adultos com o ambiente natural e com as gerações mais velhas, detentoras do conhecimento tradicional, fica quase restrito aos finais de semana e feriados. As mulheres constituem um elo entre as duas gerações, por suas atividades domésticas mais próximas ao campo. A principal atividade é a prestação de serviços, mas a agricultura familiar tradicional ainda é a única fonte de renda e sobrevivência de muitos moradores.

Situada a poucos quilômetros do centro de Abreu e Lima, encontra-se a comunidade Inhamã, a qual ocupa uma área de aproximadamente 157.800 ha e está representada por parcelas de assentamentos rurais realizados em área circundada por antigas e históricas propriedades. Por situarse muito próxima à capital, processos como a urbanização e êxodo rural têm sido acelerados e os moradores têm como principal atividade a prestação de serviços em indústrias e empresas. A comunidade dispõe de um posto municipal de saúde pública onde são distribuídos medicamentos para os problemas mais comuns, como hipertensão, colesterol elevado e diabetes. Dispõe, também, da Associação de Moradores e Produtores Rurais de Inhamã/Abreu e Lima (AMPRIAL), que está ligada a vários programas de assistência governamental, incluindo incentivo à produção rural e agricultura de subsistência. As casas são de alvenaria, quase todas dispondo de água encanada e sistema de esgoto, e possuem quintais e jardins onde espécies medicinais podem ser encontradas.

Inicialmente foram contatados os representantes da AMPRIAL, para esclarecimento dos objetivos do trabalho e para obter permissão dos membros da comunidade para o desenvolvimento da pesquisa, com assinatura do Termo de Consentimento Livre e Esclarecido. As informações sobre plantas medicinais foram obtidas em oito meses de trabalho de campo, por meio de entrevistas semiestruturadas junto a 75 informantes (52 mulheres e 23 homens), representando $90 \%$ das residências existentes em Inhamã. Além das entrevistas, adotou-se a turnê guiada para observação de jardins, quintais, ruas e roçados, onde se estabeleceu diálogo com os

Rev. Bras. PI. Med., Campinas, v.16, n.3, supl. I, p.721-730, 2014. 
moradores e proprietários sobre as plantas, seus usos e cultivo e realizou-se coleta das amostras do material botânico para identificação das espécies.

As plantas citadas pelos informantes foram coletadas e herborizadas e as identificações foram confirmadas por taxonomistas da Empresa de Pesquisa Agropecuária de Pernambuco. Exsicatas foram tombadas nos herbários IPA e UFP.

A importância relativa (IR) de cada espécie, cujo valor máximo é 2 (Bennett \& Prance, 2000), foi estimada empregando-se a fórmula IR=NSC+NP, onde $\mathrm{NSC}=$ número de sistemas corporais que são tratados pela espécie dividido pelo número de sistemas corporais tratados pela espécie mais versátil e NP= número de propriedades atribuídas à espécie dividido pelo número total de propriedades atribuídas à espécie mais versátil.

Considerando as indicações terapêuticas, as espécies foram enquadradas nos diferentes tipos de transtornos relacionados com os sistemas corporais reconhecidos pela Organização Mundial de Saúde (OMS, 2008).

Após a conclusão do estudo, dados disponíveis na literatura científica foram associados às espécies mais citadas pelos informantes e um texto com estas informações foi entregue à liderança da AMPRIAL, para posterior repasse aos moradores.

\section{RESULTADOS E DISCUSSÃO}

Metade dos 75 entrevistados encontrase na faixa etária de 29 a 56 anos e a principal atividade econômica é a prestação de serviços, com apenas $19 \%$ constituído por agricultores. O nível de escolaridade é muito baixo e apenas $10,7 \%$ têm o ensino médio completo.

A maioria dos indivíduos abordados (64\%) citou entre uma e dez espécies vegetais com uso terapêutico e poucos entrevistados conheciam mais de 20 plantas medicinais. Os poucos indivíduos que citaram mais de 30 espécies (5\%) são homens e mulheres na faixa de 43 a 70 anos e a maioria cultiva plantas medicinais.

Apesar da proximidade com a capital, do crescente êxodo rural e da urbanização de lotes de terra, dentre as espécies citadas pelos informantes pouco mais da metade (54\%) são cultivadas no local pelo próprio usuário, o que diferencia esta comunidade de outras localizadas junto a centros urbanos. Apenas 10 pessoas identificaram-se como agricultores, porém cerca de $30 \%$ dos entrevistados cultivam as espécies medicinais em quintais, em roçados ou em associação destes. A maioria das espécies é herbácea $(38,8 \%)$, seguindo-se as arbustivas $(20,4 \%)$, sendo em menor número as arbóreas. A predominância de herbáceas é frequentemente mencionada em pesquisas sobre plantas medicinais realizadas junto a comunidades urbanas, rurais ou tradicionais, como as estudadas por Silva \& Andrade $(1998,2004,2005)$ em Pernambuco. Nas turnês guiadas realizadas em Inhamã, as herbáceas mais encontradas nos quintais foram Mentha villosa Becker (hortelã), P. barbatus e Chenopodium ambrosioides L. (mastruz). Os portes arbóreo e subarbustivo estão mais associados aos roçados, muitas vezes pela sombra gerada por espécies como o juazeiro (Ziziphus joazeiro Mart.), o cajueiro (A. occidentale) e a mangueira (Mangifera indica L.).

São conhecidas como medicinais na comunidade Inhamã 155 espécies, distribuídas em 112 gêneros e 59 famílias (Tabela 1). Destacamse pelo número de espécies as Fabaceae (14), Lamiaceae (11), Asteraceae (9), Euphorbiaceae (7) e Solanaceae (7). Apiaceae e Arecaceae tiveram quatro espécies citadas, enquanto três espécies foram relatadas para as famílias Anacardiaceae, Lauraceae, Moraceae, Poaceae, Rutaceae e Zingiberaceae. Quase a metade (25) das famílias está representada por apenas uma ou duas espécies, porém algumas se destacam pelo número de citações e indicações terapêuticas, como Amaryllidaceae, representada pelo alho (Allium sativum $L$.) e Caricaceae, representada pelo mamão (Carica papaya L.).

As famílias que apresentaram maior número de espécies também se destacam em estudos realizados em diferentes regiões do Brasil, sinalizando para uma provável eficácia de suas espécies na produção de compostos bioativos de ação medicinal (Fuck et al., 2005; Pinto et al., 2006; Teixeira \& Melo, 2006; Pilla et al., 2006).

As espécies com maior número de citações foram $M$. villosa (hortelã da folha miúda, 65\%), C. citratus (capim santo, 49\%), C. ambrosioides (mastruz, 49\%) e Plectranthus amboinicus (Lour.) Spreng. (hortelã da folha grande, 45\%). Embora nem sempre as indicações terapêuticas sejam as mesmas, estas espécies são também as mais citadas em estudos desenvolvidos em diferentes regiões do país, como é observado no Nordeste (Pinto et al., 2006; Teixeira \& Melo, 2006), Sudeste (Pilla et al., 2006) e Sul (Fuck et al., 2005).

As folhas foram as partes das plantas mais utilizadas, seguidas de cascas do tronco, flores, raízes, sementes e látex, preparadas na maioria das vezes como chá, obtido por infusão ou decocção (Tabela 1). Algumas plantas são vendidas sob a forma de lambedores e garrafadas (feitos a partir da mistura de várias plantas) ou como pó (Z. joazeiro e P. edulis).

Devido às características sócio-culturais e econômicas da comunidade estudada, são muito frequentes transtornos do sistema digestório

Rev. Bras. PI. Med., Campinas, v.16, n.3, supl. I, p.721-730, 2014. 
TABELA 1. Plantas medicinais citadas pelos entrevistados da comunidade rural de Inhamã, município de Abreu e Lima, PE, Brasil.

\begin{tabular}{|c|c|c|c|}
\hline $\begin{array}{l}\text { Nome vulgar e } \\
\text { Fonte }\end{array}$ & Espécie & Indicação & Partes/preparo \\
\hline Abacate ${ }^{1}$ & Persea americana Mill. & $\begin{array}{l}\text { Afecções renais; inflamações; infecção urinária; } \\
\text { indigestão. }\end{array}$ & F: inf.; dec. \\
\hline Abacaxi ${ }^{1}$ & Ananas sativus Schult. f. & Catarro no peito; asma; inflamação dos brônquios. & Fr: lamb. \\
\hline Açaí ${ }^{1}$ & Euterpe oleracea Mart. & Anemia. & Fr: suco. \\
\hline 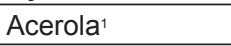 & Malpighia emarginata DC. & Tosse; catarro no peito; gripe; asma. & Fr: lamb.; suco. \\
\hline Acônito ${ }^{1}$ & $\begin{array}{l}\text { Pfaffia glomerata (Spreng.) } \\
\text { Pederson }\end{array}$ & Febre; dores em geral. & F: inf.; dec. \\
\hline Agrião $^{2}$ & Nasturtium officinale R.Br. & Tosse; catarro no peito; gripe. & F: dec.; lamb. \\
\hline Alcachofra ${ }^{1}$ & $\begin{array}{l}\text { Vernonia condensata } \\
\text { Baker }\end{array}$ & $\begin{array}{l}\text { Afecções do fígado; dores ou cólicas abdominais; } \\
\text { indigestão; obesidade. }\end{array}$ & F: inf.; dec.; suco. \\
\hline Alecrim $^{3}$ & Rosmarinus officinalis L. & Sinusite; dores corporais. & F:dec.; culinária. \\
\hline $\begin{array}{l}\text { Alfavaca; } \\
\text { alfavaca de } \\
\text { caboclo }^{1}\end{array}$ & Ocimum gratissimum $\mathrm{L}$. & $\begin{array}{l}\text { Catarro no peito; febre; sinusite; dores de ouvido } \\
\text { afecções dos olhos; diarreia; cólicas menstruais; } \\
\text { transtornos do sistema nervoso; afecções gênito- } \\
\text { urinárias. }\end{array}$ & $\begin{array}{l}\text { F: inf.; dec.; lamb.; } \\
\text { suco. }\end{array}$ \\
\hline Alho; alho roxo ${ }^{3}$ & Allium sativum $\mathrm{L}$. & $\begin{array}{l}\text { Hipertensão; gripe; hemorroidas; afecções parasitárias; } \\
\text { colesterol alto; afecções do aparelho reprodutor } \\
\text { feminino. }\end{array}$ & B:inf.;lamb.; mac.; sup. \\
\hline Alpiste $^{3}$ & Phalaris canarienses L. & Afecções renais. & S: dec. \\
\hline Amora branca $^{3}$ & Morus alba L. & Hipertensão. & F: inf. \\
\hline Anador $^{1}$ & Justicia gendarussa Burm. f. & Febre; gripe; cólicas menstruais, dores abdominais. & F: inf.; dec. \\
\hline Aniz-estrelado $^{3}$ & Illicium verum Hook f. & Má digestão. & S: dec. \\
\hline Araçá $^{3}$ & Psidium araça Radd. & Má digestão. & F: dec. \\
\hline Aroeira $^{1}$ & Schinus terebinthifolius Raddi & Tosse; gripe; inflamações em geral; cicatrizante. & CT: dec.; ext. \\
\hline Arroz chocho $^{3}$ & Não identificada & Inflamações em geral. & R: dec. \\
\hline Arruda ${ }^{1}$ & Ruta graveolens L. & $\begin{array}{l}\text { Dores de ouvido; afecções parasitárias; cólicas } \\
\text { menstruais; dores corporais. }\end{array}$ & F: inf.; dec.; sumo. \\
\hline Artemísia $^{1}$ & Artemisia vulgaris $\mathrm{L}$. & $\begin{array}{l}\text { Afecções renais; hipertensão; afecções do fígado; } \\
\text { stress; ansiedade. }\end{array}$ & F: inf. \\
\hline Aticum $^{2}$ & Annona sp. & Dores de cabeça. & F:aquecida u.t. \\
\hline Atipim 1 & Petiveria alliacea L. & Dores corporais; reumatismo. & F e R: F; ext. \\
\hline Aveloz $^{2}$ & Euphorbia tirucalli $\mathrm{L}$. & Verruga. & L: u. t. \\
\hline Azeitona roxa ${ }^{2}$ & Syzygium cumini Skeels & \begin{tabular}{|l|} 
Colesterol alto. \\
\end{tabular} & F: dec. \\
\hline Babosa $^{1}$ & Aloe vera (L.) Burm. f. & $\begin{array}{l}\text { Hemorróidas;cortes, feridas; inflamações; calvície; } \\
\text { úlceras; gastrite. }\end{array}$ & F:sumo (u.t.); sup. \\
\hline Banana $^{1}$ & Musa paradisiaca L. & $\begin{array}{l}\text { Tosse; bronquite; hemorroidas; afecções parasitárias; } \\
\text { afecções renais; hipertensão; hemorragia de } \\
\text { ferimentos; tuberculose; coqueluche. }\end{array}$ & $\begin{array}{l}\text { Fl e R: lamb. } \\
\text { L: u.t. } \\
\text { Fr: ingestão in natura. }\end{array}$ \\
\hline Barba de bode ${ }^{2}$ & Indigofera sp. & Doenças venéreas. & R: dec. \\
\hline Barbatimão $^{1}$ & Stryphnodendron sp. & $\begin{array}{l}\text { Feridas abertas; inflamações em geral; afecções } \\
\text { cutâneas. }\end{array}$ & CT: dec. \\
\hline Batata de purga ${ }^{3}$ & Operculina alata (Ham.) Urb. & $\begin{array}{l}\text { Prisão de ventre; infecções intestinais; sinusite; } \\
\text { derrame. }\end{array}$ & S: inf. \\
\hline 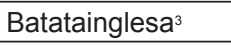 & Solanum tuberosum L. & \begin{tabular}{|l|} 
Dores de cabeça; gastrite. \\
\end{tabular} & R: u. t. \\
\hline Bem-me-quer $^{1}$ & Sphagneticola trilobata (L.) Prusk & Inflamações em geral. & P: u. t. \\
\hline Berinjela $^{3}$ & Solanum melongena L. & Colesterol alto. & Fr: suco. \\
\hline Beterraba $^{3}$ & Beta vulgaris $\mathrm{L}$. & Tosse;catarro ; infecções. & R: lamb. \\
\hline Boldo do Chile ${ }^{3}$ & Pneumus boldus Molina & $\begin{array}{l}\text { Diarreia; indigestão; dores ou cólicas abdominais; } \\
\text { afecções do fígado. }\end{array}$ & F: inf.; dec. \\
\hline Boldo da Índia1 & Plectranthus neochilus Schlechter & Afecções do fígado; indigestão; cólicas em geral. & F: inf.; dec. \\
\hline $\begin{array}{l}\text { Boldo do Pará ou } \\
\text { do mato' }\end{array}$ & Plectranthus barbatus Andrews & $\begin{array}{l}\text { Afecções do fígado; indigestão; gastrite; dores } \\
\text { corporais. }\end{array}$ & F: inf. \\
\hline Bredo $^{2}$ & Talinum racemosum (L.) Rohrb. & Desnutrição infantil (carência de cálcio). & $\mathrm{F}$ : inf. \\
\hline Café beirão1 & Senna alata (L.) Roxb. & Sinusite; tosse; micoses; inflamação dos brônquios. & F: inf. \\
\hline Caju roxo $^{1}$ & Anacardium occidentale L. & Inflamações em geral; derrame; feridas. & CT: ext. \\
\hline
\end{tabular}


TABELA 1. Plantas medicinais citadas pelos entrevistados da comunidade rural de Inhamã, município de Abreu e Lima, PE, Brasil.

continuação...

\begin{tabular}{|c|c|c|c|}
\hline Camomila $^{3}$ & Matricaria chamomilla L. & Transtornos do sistema nervoso. & FI: dec. \\
\hline $\begin{array}{l}\text { Cana caiana; } \\
\text { cana } 1\end{array}$ & Saccharum sp. & Anemia; hipertensão. & F:inf.;lamb.;sumo. \\
\hline Cana de macaco 1 & Costus spicatus (Jacq.) Sw. & Afecções renais; inflamações da coluna. & P: inf. \\
\hline Canela ${ }^{1}$ & Cinnamomum zeylanicum Breun. & $\begin{array}{l}\text { Transt. do sistema nervoso; catarro no peito; } \\
\text { hipertensão; indigestão; vômitos. }\end{array}$ & F: dec. \\
\hline Cânfor ${ }^{2}$ & Não identificada & Tosse. & R: dec. \\
\hline Capeba $^{1}$ & Begonia sp. & $\begin{array}{l}\text { Anemia; afecções do fígado; indigestão; afecções } \\
\text { renais. }\end{array}$ & F: dec. \\
\hline Capim santo ${ }^{1}$ & Cymbopogon citratus (DC.) Stapf & $\begin{array}{l}\text { Transtornos do sistema nervoso; anemia; afecções } \\
\text { parasitárias; hipertensão; diarreia; indigestão; dores } \\
\text { ou cólicas abdominais. }\end{array}$ & F: inf.; dec. \\
\hline Carambola ${ }^{1}$ & Averrhoa carambola L. & $\begin{array}{l}\text { Transtornos do sistema nervoso; cólicas; afecções } \\
\text { do fígado. }\end{array}$ & F: inf.; suco. \\
\hline $\begin{array}{l}\text { Cardeiro; } \\
\text { mandacaru }{ }^{1}\end{array}$ & Cereus jamacaru DC. & $\begin{array}{l}\text { Afecções renais; diabetes; inflamações da coluna; } \\
\text { afeç̧ões parasitárias. }\end{array}$ & Fr: dec. \\
\hline Cardo santo $^{1}$ & Argemone mexicana L & Derrame. & S: dec. \\
\hline Carrapateira $^{2}$ & Ricinus communis L. & Dores de cabeça; catapora. & Fr: azeite. \\
\hline $\begin{array}{l}\text { Catingueira; cating. } \\
\text { rasteir }^{3}\end{array}$ & Não identificada & Derrame; sinusite; impotência sexual masculina. & S: inf. \\
\hline Cebolinha do mato $^{1}$ & Eleutherine bulbosa (Mill.) Urb. & Tosse; catarro no peito. & B: lamb. \\
\hline Cenoura $^{3}$ & Daucus carota L. & Catarro no peito; infecções. & R: lamb. \\
\hline Cereja selvagem $^{1}$ & Bunchosia armeniaca DC. & Anemia. & Fr: ingestão in natura. \\
\hline Chambá ${ }^{1}$ & Justicia pectoralis Jacq. & Tosse; catarro; febre; gripe. & F: lamb.; inf.; dec. \\
\hline Chanana $^{2}$ & Turnera ulmifolia L. & Tosse. & $\begin{array}{l}\text { F, FI e R: lamb.; inf.; } \\
\text { dec. }\end{array}$ \\
\hline Chuchu $^{3}$ & Sechium edule (Jacq.) Swartz. & Hipertensão. & Fr: ingestão in natura. \\
\hline Cipó de vaqueiro ${ }^{3}$ & Cardiospermum halicacabum L. & Contusões. & P: dec. \\
\hline $\begin{array}{l}\text { Coco amarelo/ } \\
\text { verde }^{1}\end{array}$ & Cocos nucifera L. & $\begin{array}{l}\text { Diarreia; desidratação; micoses; anemia; hipertensão; } \\
\text { inflamação do olho. }\end{array}$ & Fr: dec. \\
\hline Colônia ${ }^{1}$ & $\begin{array}{l}\text { Alpinia zerumbet (Pers.) B. L. Burtt. } \\
\text { \& R. M. Sm. }\end{array}$ & $\begin{array}{l}\text { Tosse; catarro no peito; febre; dores em geral; } \\
\text { ansiedade. }\end{array}$ & F: inf.; dec. \\
\hline Confrei $^{1}$ & \begin{tabular}{|l|l|} 
Symphytum officinale L. & \\
\end{tabular} & Inflamações em geral; ferimentos não cicatrizados. & F: u. t. \\
\hline Cordão de frade ${ }^{2}$ & Leonotis nepetaefolia R. Br. & Afecções parasitárias. & I: dec. \\
\hline Coroa de frade $^{1}$ & \begin{tabular}{|l|l|} 
Não identificada \\
\end{tabular} & Afecções parasitárias. & Fr: dec. \\
\hline 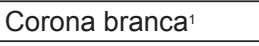 & Bryophyllum pinnatum (Lam.) Oken & Tosse; catarro no peito; bronquite; gastrite. & F:inf.;lamb.; suco. \\
\hline Corona roxa ${ }^{2}$ & Kalanchoe brasiliensis Camb. & Tosse. & F: lamb. \\
\hline Couve folha ${ }^{1}$ & Brassica oleracea L. & Gastrite. & F: suco. \\
\hline Coentro roxo 1 & Coriandrum sativum $\mathrm{L}$. & Derrame. & F: dec. \\
\hline Dendê $^{3}$ & Elaeis guineenses Jacq & Afecções cutâneas. & Fr: azeite. \\
\hline $\begin{array}{l}\text { Erva cidreira; erva } \\
\text { cidreira branca }{ }^{1}\end{array}$ & Lippia alba (Mill.) N. E. Br. & $\begin{array}{l}\text { Transt. do sistema nervoso; complicações do pré e } \\
\text { pós-parto; indigestão; menstruação atrasada; cólicas } \\
\text { menstruais; anemia;; hipertensão; enxaqueca; } \\
\text { intoxicação. }\end{array}$ & F: inf.; dec. \\
\hline Erva doce $^{3}$ & Pimpinella anisum $\mathrm{L}$. & $\begin{array}{l}\text { Náuseas; flatulência; enxaqueca; hipertensão; diarreia; } \\
\text { vômitos. }\end{array}$ & F: inf.; dec. \\
\hline Erva moura $^{1}$ & Solanum americanum Mill. & Micoses; gastrite; varizes; hemorroidas; contusões. & F: mac. \\
\hline Erva rosa ${ }^{1}$ & Não identificada & Dores do aparelho reprodutor masculino e feminino. & F: inf. \\
\hline Espinho de cigano ${ }^{1}$ & Acanthospermum hispidum DC. & Tosse; catarro no peito; bronquite; diabetes. & R: dec.; lamb. \\
\hline Fruta pão ${ }^{1}$ & $\begin{array}{l}\text { Artocarpus communis J.R. Forst. } \\
\text { \& G. Forst. }\end{array}$ & Inchaço das pernas. & F: dec. \\
\hline Gaigirú1 & Phaseolus sp. & Inflamações da coluna ou nas articulações. & F: inf. \\
\hline Gengibre $^{1}$ & Zingiber sp. & $\begin{array}{l}\text { Indigestão; tosse; inflamação da garganta; dores } \\
\text { corporais. }\end{array}$ & R: lamb.; dec. \\
\hline $\begin{array}{l}\text { Gergelim; gergilim } \\
\text { preto }^{3}\end{array}$ & Sesamum orientale L. & Sinusite; derrame. & S: inf. \\
\hline Genipapo $1^{1}$ & Genipa americana L. & Tosse; catarro; anemia; câncer. & Fr: inf.; lamb. \\
\hline
\end{tabular}

continua...

Rev. Bras. PI. Med., Campinas, v.16, n.3, supl. I, p.721-730, 2014. 
TABELA 1. Plantas medicinais citadas pelos entrevistados da comunidade rural de Inhamã, município de Abreu e Lima, PE, Brasil.

continuação...

\begin{tabular}{|c|c|c|c|}
\hline Gerimum ${ }^{1}$ & Cucurbita pepo L. & $\begin{array}{l}\text { Dores de ouvido; afecções parasitárias; afecções da } \\
\text { próstata; prisão de ventre. }\end{array}$ & FI: mac.; u. t. \\
\hline Girassol $^{3}$ & Helianthus annuus L. & Sinusite; derrame. & S: inf.; dec. \\
\hline Goiaba1 $^{1}$ & Psidium guajava L. & Perda de cálcio; indigestão; dores abdominais; diarreia. & F: inf.; dec. \\
\hline Graviola1 & Annona muricata L. & Câncer. & S: torrada. \\
\hline Guaco $^{2}$ & Mikania glomerata. Spreng. & Tosse; catarro no peito. & F: lamb. \\
\hline Hortelã grande ${ }^{1}$ & $\begin{array}{l}\text { Plectranthus amboinicus (Lour.) } \\
\text { Spreng. }\end{array}$ & $\begin{array}{l}\text { Tosse; catarro no peito; bronquite; gripe; transt. do } \\
\text { sistema nervoso. }\end{array}$ & F: inf.; dec.; lamb. \\
\hline Hortelã pequeno ${ }^{1}$ & Mentha villosa Becker & $\begin{array}{l}\text { Afecções parasitárias; cólicas menstruais; tosse; } \\
\text { gripe; indigestão; diarreia; hemorroidas; convulsão de } \\
\text { recém-nascidos. }\end{array}$ & F: inf.; lamb. \\
\hline $\begin{array}{l}\text { Imburana de } \\
\text { cheiro }^{3}\end{array}$ & $\begin{array}{l}\text { Amburana cearensis (Fr.Allem.) } \\
\text { A.C. Smith }\end{array}$ & Sinusite; derrame. & S: torrada; inf. \\
\hline Insulina ${ }^{1}$ & $\begin{array}{l}\text { Cissus verticillata (L.) Nicolson \& } \\
\text { C. E. Jarvis }\end{array}$ & Diabetes. & F: inf. \\
\hline Jambo $^{1}$ & $\begin{array}{l}\text { Syzygium malaccense (L.) Merr. \& } \\
\text { L. M. Perry. }\end{array}$ & Gastrite. & Fr: suco. \\
\hline Jasmim $^{1}$ & Não identificada & Coqueluche. & Fl: lamb. \\
\hline Jatobá1 & Hymenaea courbaril L. & Feridas não cicatrizadas. & F: lamb; dec. \\
\hline Juá1 & Ziziphus joazeiro Mart. & Catarro no peito; gripe; cáries; caspa. & CT: pó. \\
\hline Jucá ${ }^{3}$ & Caesalpinia ferrea Mart. & Contusões. & Fr: dec. \\
\hline Jurubeba ${ }^{1}$ & Solanum paniculatum $\mathrm{L}$. & $\begin{array}{l}\text { Bronquite, catarro no peito; anemia; afecções do } \\
\text { fígado. }\end{array}$ & F: dec; suco. \\
\hline Laranja $^{1}$ & Citrus sinensis Osbeck. & $\begin{array}{l}\text { Colesterol alto; diarreia; transtornos do sistema } \\
\text { nervoso. }\end{array}$ & F: suco; inf. \\
\hline Liamba $^{2}$ & Vitex agnus-castus L. & Dor de dente; inflamações & F: dec. \\
\hline Liga osso $^{3}$ & Dorstenia sp. & Fraturas ósseas. & F: dec. \\
\hline Limão $^{1}$ & Citrus limon (L.) Burn. f. & Colesterol alto; odores nas axilas; diabetes. & Fr: suco; inf. \\
\hline Língua de sapo & Peperomia pellucida (L.) H. B. K. & Colesterol alto. & P: inf. \\
\hline Louro $^{1}$ & Ocimum sp. & Gastrite; obesidade. & F: dec. \\
\hline Lombrigueira $^{2}$ & Spigelia anthelmia L. & Afecções parasitárias. & F: inf. \\
\hline Maçã ${ }^{3}$ & Pyrus malus L. & Hipertensão. & Fr: suco. \\
\hline Macassá & $\begin{array}{l}\text { Aeolanthus suaveolens Mart. ex } \\
\text { Spreng }\end{array}$ & Dores de ouvido; dores corporais. & F: sumo, ingerir. \\
\hline Macaíba $^{2}$ & Acrocomia intumescens Drude & Coqueluche; tuberculose; pneumonia. & Fr: lamb. \\
\hline $\begin{array}{l}\text { Macaxeira; } \\
\text { mandioca }{ }^{1}\end{array}$ & Manihot esculenta Crantz. & Afecções dos olhos; desnutrição infantil. & F: farinha. \\
\hline Malva rosa ${ }^{1}$ & Urena lobata L. & Sinusite; dores corporais. & F: inf.; dec. \\
\hline Malva santa $^{3}$ & Malva sp. & Derrame. & F: inf. \\
\hline Malvaísco² & Piper marginatum Jacq. & Hemorroidas. & F: inf. \\
\hline $\begin{array}{l}\text { Mamão verde / } \\
\text { roxo }^{1}\end{array}$ & Carica papaya L. & Prisão de ventre; asma; indigestão; diarreia; verruga. & F:dec.;u. t.; sumo. \\
\hline 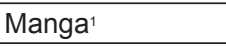 & Mangifera indica L. & Asma; tosse; catarro no peito; bronquite. & Fr: lamb. \\
\hline Manjericão' ${ }^{1}$ & Ocimum sp. & $\begin{array}{l}\text { Dores de ouvido ou corporais; afecções dos olhos; } \\
\text { febre; bronquite; dores corporais. }\end{array}$ & F: dec.; mac. \\
\hline $\begin{array}{l}\text { Manjerioba; } \\
\text { manjeroba }^{2}\end{array}$ & Senna occidentale L. & Bronquite; tosse; sinusite. & $\begin{array}{l}\text { S: inf. } \\
\text { R: dec. }\end{array}$ \\
\hline $\begin{array}{l}\text { Manjericão } \\
\text { miúdo }^{1}\end{array}$ & Ocimum basilicum $\mathrm{L}$. & Afecções dos olhos. & F: suco; u.t. \\
\hline Maracujá ${ }^{1}$ & Passiflora edulis Sims. & $\begin{array}{l}\text { Tosse; hipertensão; diabetes; transtornos do sistema } \\
\text { nervoso. }\end{array}$ & $\begin{array}{l}\text { Fr: pó; lamb. } \\
\text { S: lamb. } \\
\text { F: inf; dec. }\end{array}$ \\
\hline Mastruz ${ }^{1}$ & Chenopodium ambrosioides $\mathrm{L}$. & $\begin{array}{l}\text { Gripe; catarro no peito; afecções parasitárias; } \\
\text { tuberculose; bronquite. }\end{array}$ & $\begin{array}{l}\text { F: inf.; dec.; lamb.; } \\
\text { suco. }\end{array}$ \\
\hline Mata pasto ${ }^{3}$ & $\begin{array}{l}\text { Senna obtusifolia (L.) H.S. Irwin \& } \\
\text { Barnbey }\end{array}$ & Sinusite; derrame. & S: inf.; torrada. \\
\hline Melancia $^{3}$ & Citrullus vulgaris Schrad. & Derrame; febre; prisão de ventre; impotência sexual. & S: dec. \\
\hline
\end{tabular}


TABELA 1. Plantas medicinais citadas pelos entrevistados da comunidade rural de Inhamã, município de Abreu e Lima, PE, Brasil.

continuação...

\begin{tabular}{|c|c|c|c|}
\hline $\begin{array}{l}\text { Melão de buriatã/ } \\
\text { São Caetano }\end{array}$ & Momordica charantia L. & Hemorróidas. & $\begin{array}{l}\text { F: dec.; mac. } \\
\text { FI: dec.; mac. }\end{array}$ \\
\hline Meracilina ${ }^{1}$ & Graptophyllum pictum Griff. & Inflamações em geral. & F: inf. \\
\hline Mirra $^{1}$ & Não identificada & Dores corporais. & F: inf. \\
\hline Moela de galinha ${ }^{1}$ & Polyscias balfouriana L. H. Bailey & Indigestão; diarreia. & F: inf; dec. \\
\hline Mostarda $^{3}$ & Não identificada & Sinusite; derrame. & S: inf.; torrada. \\
\hline Mulungú1 & Erythina velutina Willd. & Transtornos do sistema nervoso; sinusite; derrame. & $\begin{array}{l}\text { F: dec.CT: dec. } \\
\text { S: inf.; torrada. }\end{array}$ \\
\hline Mussambê ${ }^{2}$ & Cleome spinosa Jacq. & Tosse; gripe; bronquite. & F, FI, R:inf.; dec \\
\hline Orelha de sapo ${ }^{2}$ & Não identificada & Hipertensão. & F: inf. \\
\hline Parreira $^{3}$ & Vitis vinifera L. & Tosse; gripe. & C: dec. \\
\hline $\begin{array}{l}\text { Pata de vaca; crote } \\
\text { coité }^{2}\end{array}$ & Bauhinia sp. & Indigestão; diabetes; afecções renais. & F: inf. \\
\hline Pega pinto ${ }^{2}$ & Boerhavia diffusa $\mathrm{L}$. & $\begin{array}{l}\text { Afecções renais; afecções da próstata; infec. do sist. } \\
\text { reprod. }\end{array}$ & F e R: inf.; dec. \\
\hline Pepino $^{3}$ & Cucumis sativus L. & Hipertensão; colesterol alto. & Fr: lamb.; suco. \\
\hline Pequí3 ${ }^{3}$ & Cariocarsp. & Inflamações em geral. & Fr: óleo; u.t. \\
\hline Pimenta $1^{1}$ & Capsicum sp. & Dor de dente; furúnculos. & F: dec.; catp. \\
\hline Pimentão $^{3}$ & Capsicum annuum L. & Anemia. & Fr: ingestão. \\
\hline Pindaíba $^{3}$ & Não identificada & Sinusite; derrame. & S: inf.; torrada. \\
\hline Pinhãobranco² & Jatropha pohliana M. & Asma. & F: suco. \\
\hline Pinhão roxo² & Jatropha gossypiifolia L. & Tosse; catarro no peito. & S: lamb. \\
\hline Piri piri² & Averrhoa bilimbi L. & Diabetes. & Fr: inf. \\
\hline 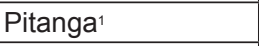 & Eugenia pitanga Arech. & Indigestão; diarreia; cólica intestinal. & F: inf.; dec. \\
\hline $\begin{array}{l}\text { Quebra pedra } \\
\text { branco }^{2}\end{array}$ & Phyllanthus niruri L. & $\begin{array}{l}\text { Afecções renais; infecção urinária; inflamações da } \\
\text { coluna; menstruação atrasada; cólicas menstruais. }\end{array}$ & F e R: dec. \\
\hline Quebra pedra roxo 1 & Chamaecyce thymifolia (L.) Mill. & Afecções renais; Infecção urinária. & P: inf.; dec. \\
\hline Quiabo' $^{1}$ & Hibiscus esculentus L. & Asma. & Fr: lamb. \\
\hline Quixaba $^{3}$ & $\begin{array}{l}\text { Sideroxylon obtusifolium (Roem. e } \\
\text { Schult.) }\end{array}$ & Inflamações da coluna; contusões. & CT: dec. \\
\hline Rabo de raposa ${ }^{2}$ & $\begin{array}{l}\text { Conyza sumatrensis (Ritz.) E. } \\
\text { Walker }\end{array}$ & Micoses. & F: u. t. \\
\hline Rama de St ${ }^{\mathrm{a}}$ Luzia $^{2}$ & Commelina benghalensis L. & Afecções dos olhos. & Fl: sumo. \\
\hline Romã $^{1}$ & Punica granatum $\mathrm{L}$. & $\begin{array}{l}\text { Tosse; cólicas menstruais; afecções dos olhos; gastrite; } \\
\text { inflamação da garganta. }\end{array}$ & $\begin{array}{l}\text { Fr: inf.; dec.; lamb.; } \\
\text { suco. }\end{array}$ \\
\hline Sabugo $^{1}$ & Sambucus australis Cham. & Febre; sarampo; afecções renais; tosse; bronquite. & FI: inf.; dec.; lamb. \\
\hline Salsa $^{1}$ & Não identificada & Micoses. & F: u. t. \\
\hline Sapotí1 & Achras sapota L. & Anemia. & Fr: suco. \\
\hline $\begin{array}{l}\text { Tamarina; } \\
\text { tamarindo }^{2}\end{array}$ & Tamarindus sp. & Tosse;catarro no peito. & Fr: lamb. \\
\hline Tomate $^{3}$ & Lycopersicon esculentum Mill. & Furúnculos. & Fr: u. t. \\
\hline Trapiá $^{1}$ & \begin{tabular}{|l|l} 
Não identificada \\
\end{tabular} & Sinusite. & F: catp. \\
\hline Unha de gato ${ }^{2}$ & $\begin{array}{l}\text { Mimosa polydactyla Humb. \& } \\
\text { Bonpl. }\end{array}$ & Afecções da próstata; infecção urinária. & R:dec. \\
\hline Urinama $^{2}$ & \begin{tabular}{|l|l|} 
Zornia diphyla (L.) Pers. \\
\end{tabular} & Afecções renais. & R: dec. \\
\hline Urtiga branca ${ }^{1}$ & Não identificada & Inflamações em geral; dor de dente; colesterol alto. & R: dec. \\
\hline Vassoura de botão² & Borreria verticillata (L.) G. Mey & $\begin{array}{l}\text { Inflamações; afecções da próstata; hemorroidas; infec. } \\
\text { urinárias; doenças venéreas. }\end{array}$ & R: dec. \\
\hline Vergamota $^{1}$ & Não identificada & Cólicas menstruais; dores corporais. & F: inf.; dec. \\
\hline Vick' $^{1}$ & Mentha sp. & Tosse; gripe; sinusite; afecções parasitárias. & F: inf.; dec.; lamb. \\
\hline Zezinho $^{2}$ & Pilea hyalina Fenzl. & Colesterol alto. & P: inf. \\
\hline
\end{tabular}

1 - cultivada; 2 - não cultivada; 3 - comprada. Partes da planta: raiz (R); caule (C); folha (F); inflorescência (I); flor (FI); fruto (Fr); semente $(\mathrm{S})$; casca do tronco $(\mathrm{CT})$; látex (L); bulbo (B); planta inteira (P). Preparo: infusão (inf.); decocção (dec.); lambedor (lamb.); maceração (mac.); supositório (sup.); extrato alcoólico (ext.); uso tópico (u.t.); cataplasma (catp.) 
assim como elevada incidência de parasitoses, além das gripes e resfriados que acometem qualquer agrupamento humano. Assim sendo, os tipos de uso de plantas medicinais com maior número de citações foram relacionados a transtornos do sistema respiratório (TSR), com 46 espécies citadas, destacando-se as Fabaceae e Lamiaceae, representadas por Senna occidentale L. (mangerioba) e R. officinalis (alecrim), respectivamente; trinta espécies foram indicadas como antitussígenos e 20 como expectorantes. Para tratamento de afecções não definidas (AND), foram citadas 77 espécies, pertencentes principalmente às famílias Lamiaceae, Asteraceae e Fabaceae, representadas por O. gratissimum (alfavaca), Artemisia vulgaris L. (artemísia) e Stryphnodendron sp. (barbatimão), respectivamente. Nos transtornos do sistema gastrintestinal (TSGI) são utilizadas 35 espécies, pertencentes principalmente às famílias Lamiaceae, representada por P. barbatus, o boldo do mato, e Myrtaceae, representada por goiaba (Psidium guajava L.) e pitanga (Eugenia pitanga Arech.) (Figura 1).

Micoses superficiais são frequentes em comunidades de baixa renda, porém plantas com indicação para tratá-las não são comumente incluidas nas listas de espécies de estudos etnobotânicos. Em Inhamã, a erva-moura (Solanum americanum Mill.), o café-beirão (Senna alata (L.) Roxb.), o coqueiro (Cocos nucifera L.) e o rabo de raposa (Conyza sumatrensis (Ritz) E. Walker) foram indicados para o tratamento de micoses. Pereira et al. (2005) e Silva \& Andrade (2013) indicam o uso de uma outra espécie do gênero, Conyza bonariensis (L.) Cronquist, também conhecida como rabo de raposa, para tratamento de micoses superficiais. Oliveira et al. (2006) comentam que a alcoolatura de C. bonariensis tem sido prescrita pelos médicos do Programa Saúde da Família do Município de João Pessoa, Paraíba, para o tratamento de dermatoses. Dentre as espécies listadas na Tabela 1 algumas foram pesquisadas do ponto de vista fitoquímico e farmacológico, com ação farmacológica comprovada, como C. citratus, Lippia alba (Mill.) N. E. Br. e P. barbatus (Vale et al., 2002; Gazola et al., 2004; Costa, 2006). Outras, como Jatropha gossypiifolia L., apesar de terem ação medicinal comprovada, devem ser reavaliadas por apresentarem risco de toxidade crônica, como apontam Mariz et al. (2010). Chenopodium ambrosioides é mencionada em vários estudos sobre plantas medicinais como uma das principais espécies citadas por entrevistados, indicada como antiinflamatória, peitoral, estomáquica, antituberculosa, béquica e vulnerária, abrangendo transtornos relacionados com diferentes sistemas corporais. O suco integral desta planta costuma ser aplicado localmente nos casos de contusão e suas folhas - secas ou frescas - são também usadas em comunidades rurais como repelentes de piolhos, pulgas e carrapatos. Seu uso popular é muito antigo e a espécie está inscrita na Farmacopéia

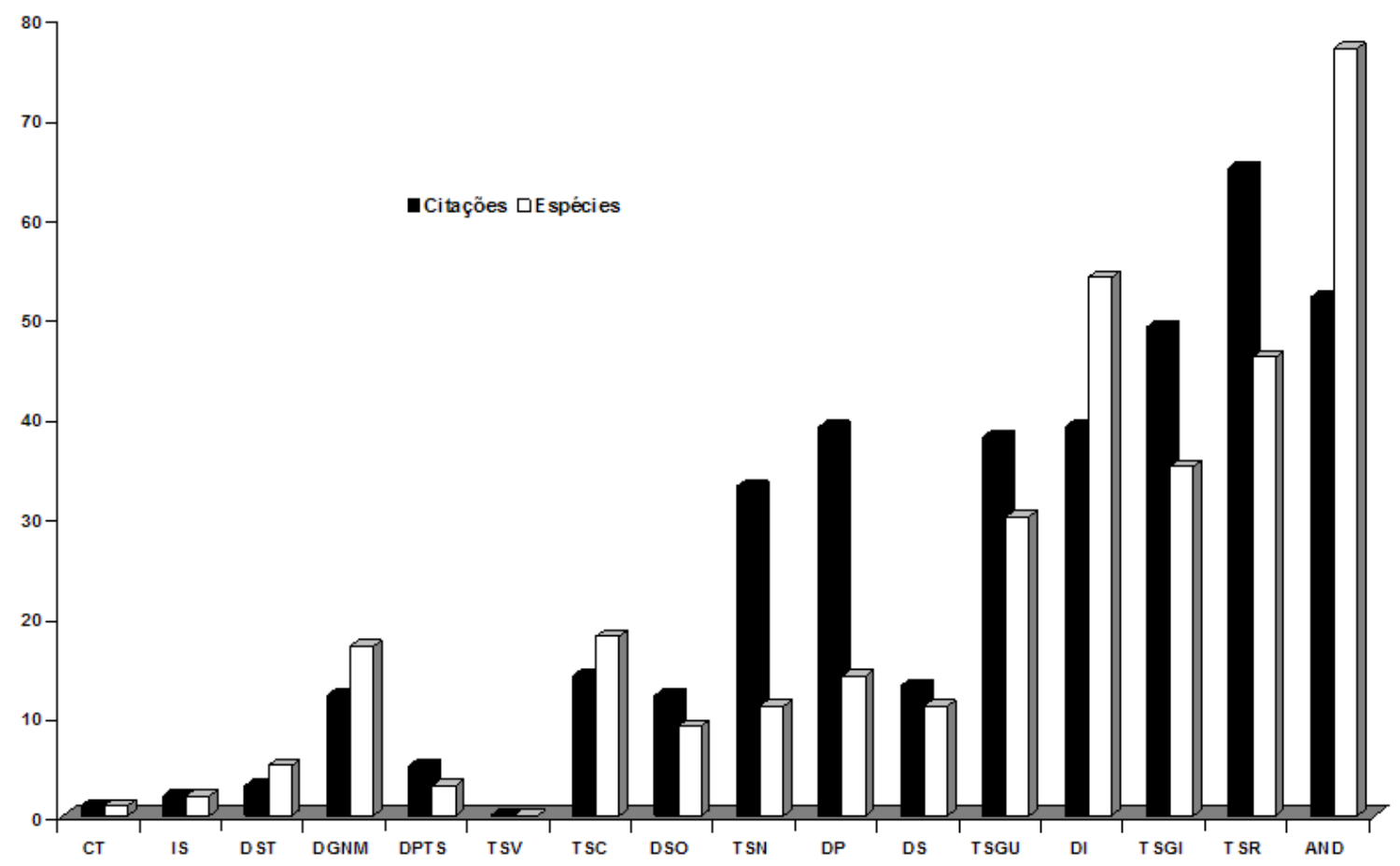

FIGURA 1. Número de citações e número de espécies citadas por moradores da comunidade Inhamã para diferentes tipos de transtornos enquadrados nos sistemas corporais reconhecidos pela Organização Mundial de Saúde (OMS, 2008). 
TABELA 2. Importância relativa das espécies medicinais com maior versatilidade de uso na comunidade de Inhamã, município de Abreu e Lima, Zona da Mata Norte de Pernambuco.

\begin{tabular}{lc}
\hline \multicolumn{1}{c}{ Espécie } & Importância relativa \\
\hline Ocimum gratissimum L. & 2,0 \\
Lippia alba (Mill.) N. E. Br. & 1,6 \\
Mentha villosa Becker & 1,6 \\
Musa paradisiaca L. & 1,5 \\
Plectranthus amboinicus (Lour.) Spreng. & 1,5 \\
Schinus terebinthifolius Raddi & 1,4 \\
Cocos nucifera L. & 1,4 \\
Alpinia zerumbet (Pers.) B. L. Burtt. \& R. M. Sm. & 1,4 \\
Punica granatum L. & 1,3 \\
Chenopodium ambrosioides L. & 1,3 \\
Pimpinella anisum L. & 1,3 \\
\hline
\end{tabular}

Brasileira I (1959), onde consta como parte usada a essência obtida dos ramos floridos e frutificados, devendo conter, no máximo, $80 \%$ de ascaridol. O óleo essencial, associado ao colódio, é usado para tratamento local da helmintíase migrante (larva migrans).

Ocimum gratissimum alcançou o valor máximo de importância relativa entre as espécies citadas como medicinais pelos informantes de Inhamã, sendo a mais versátil nesta comunidade; $L$. alba e $M$. villosa também apresentaram importância elevada, com valores de IR acima de 1,5 (Tabela 2).. A maioria das citações para tratamentos com essas plantas estava relacionada a transtornos dos sistemas respiratório, digestório e nervoso.

No Brasil, disciplinas obrigatórias relacionadas com as plantas não constam na formação rotineira dos médicos e a maior parte deles desconhece e não lança mão dos recursos terapêuticos oferecidos pelos vegetais, mesmo daqueles cientificamente testados. No presente estudo esta situação ficou bem evidente, constatando-se que apenas $11 \%$ das plantas medicinais citadas pelos moradores de Inhamã foram usadas em decorrência de prescrição médica, nenhuma dessas por profissionais do posto de saúde pública local.

Embora a indicação de medicamentos industrializados seja imprescindível para o tratamento de doenças graves ou agudas, as doenças mais comuns, como diarreias, gripes e suas consequências, poderiam ser tratadas com a prescrição de plantas medicinais devidamente certificadas. Programas de saúde associados ao uso de plantas medicinais constituiriam um incentivo governamental no uso e cultivo de espécies medicinais e contribuiriam para a manutenção do conhecimento botânico tradicional.

\section{AGRADECIMENTOS}

À Coordenadoria de Aperfeiçoamento de Pessoal de Nível Superior, CAPES, pelo financiamento parcial da pesquisa. À $\mathrm{Dr}^{\mathrm{a}}$. Rita de Cássia Araújo Pereira, curadora do Herbário IPA (Empresa Pernambucana de Pesquisa Agropecuária) e à $\mathrm{Dr}^{\mathrm{a}}$. Suzene Izidio da Silva, da Universidade Federal Rural de Pernambuco, pelo auxílio na identificação de algumas espécies vegetais. Aos moradores de Inhamã, particularmente os filiados à AMPRIAL, pela receptividade e disponibilidade em interromper seus afazeres para participar das entrevistas.

\section{REFERÊNCIA}

BENNETT, B.C.; PRANCE, G.T. Introduced plants in the indigenous pharmacopoeia of Northern South America. Economic Botany, v.54, n.1, p.90-102, 2000.

CARVALHO, M. G.; MELO, A.G.N.; ARAGÃO, C.S.F.; RAFFIN, F.N.; MOURA, T.F.A.L. Schinus terebinthifolius Raddi: chemical composition, biological properties and toxicity. Revista Brasileira de Plantas Medicinais, v.15, n.1,

Rev. Bras. PI. Med., Campinas, v.16, n.3, supl. I, p.721-730, 2014. 
p.158-169, 2013.

COSTA, M.C.C.D. Uso popular e ações farmacológicas de Plectranthus barbatus Andr. (Lamiaceae): revisão dos trabalhos publicados de 1970 a 2003. Revista Brasileira de Plantas Medicinais, v.8, n.2, p.81-88, 2006.

FARMACOPÉIA dos Estados Unidos do Brasil. 1959. 2.ed. São. Paulo: Siqueira.

FUCK, S.B.; ATHANÁZIO, J.C.; LIMA, C.B.; MING, L.C. Plantas medicinais utilizadas na medicina popular por moradores da área urbana de Bandeirantes, PR, Brasil. Semina: Ciências Agrárias, v.26, n.3, p.291-96, 2005.

GARLET, T.M.B.; IRGANG, B.E. Medicinal plants used by rural women workers in Cruz Alta, Rio Grande do Sul, Brasil. Revista Brasileira de Plantas Medicinais, v.4, n.1, p.9-18, 2001.

GAZOLA, R.; MACHADO, D.; RUGGIERO, C.; SINGI, G.; MACEDO, A.M. Lippia alba, Melissa officinalis and Cymbopogon citratus: effects of the aqueous extracts on the isolated hearts of rats. Pharmacological Research, v.50, n.5, p.477-80, 2004.

MARINHO, M.G.V.; SILVA, C.C.; ANDRADE, L.H.C. Levantamento etnobotânico de plantas medicinais em área de caatinga no município de São José de Espinharas, Paraíba, Brasil. Revista Brasileira de Plantas Medicinais, v.13, n.2, p.170-182, 2011. MARIZ, S.R.; BORGES, A.C.R.; MELO-DINIZ, M.F.F.; MEDEIROS, I.A. Possibilidades terapêuticas e risco toxicológico de Jatropha gossypiifolia L.: uma revisão narrativa. Revista Brasileira de Plantas Medicinais v.12, n.3, p. 346-357, 2010.

NEGRELLE R.B.; FORNAZZARI K.R.C. Estudo etnobotânico em duas comunidades rurais (Limeira e Ribeirão Grande) de Guaratuba (Paraná, Brasil). Revista Brasileira de Plantas Medicinais, v.9, p. 36-54, 2007.

OLIVEIRA, M.J.R.; SIMÕES, M.J.S.; SASI, C.R.R. Phytotherapy in the public health system (SUS) in the São Paulo State, Brazil. Revista Brasileira de Plantas Medicinais, v.4, n.2, p.39-41, 2006.

OLIVEIRA, R.A.G. ; LIMA, E.O.; VIEIRA, W.L.; FREIRE, K.R.L.; TRAJANO, V.N.; LIMA, I.O.; SOUZA, E.L.; TOLEDO, M.S.; SILVA-FILHO, R.N. Estudo da interferência de óleos essenciais sobre a atividade de alguns antibióticos usados na clínica. Brazilian Journal of Pharmacognosy, v.16, n.1, p.77-82, 2006.

ORGANIZAÇÃO MUNDIAL DE SAUDE-OMS. Classificação estatística internacional de doenças e problemas relacionados à saúde. São Paulo:
EDUSP. 2008.

PEREIRA, C.O. ; LIMA, E.O.; OLIVEIRA, R.A.G.; TOLEDO, M.S.; AZEVEDO, A.K.A.; GUERRA, M.F.; PEREIRA, R.C. Ethnobotanic study of medicinal plants used in dermatological disorders in João Pessoa-Paraíba, Brazil. Revista Brasileira de Plantas Medicinais, v.7, n.3, p.9-17, 2005.

PILLA, M.A.C.; AMOROZO, M.C.M.; FURLAN, A. Obtenção e uso das plantas medicinais no distrito de Martim Francisco, Município de Mogi-Mirim, SP, Brasil. Acta Botanica Brasilica, v.20, n.4, p.789802, 2006.

PINTO, E.P.P.; AMOROZO, M.C.M.; FURLAN, A. Conhecimento popular sobre plantas medicinais em comunidades rurais de Mata Atlântica Itacaré, BA, Brasil. São Paulo. Acta Botanica Brasilica, v.20, n.4, p.751-762, 2006.

PURI, S.; NAIR, P.K.R. Agroforestry research for development in Índia: 25 years of experiences of a national program. Agroforestry Systems, v.61, p.437- 52, 2004.

SILVA, A. A.; ANDRADE, L.H.C. Utilização de espécies de Asteraceae por comunidades rurais do nordeste do Brasil: relatos em Camocim de São Félix, Pernambuco. Biotemas, v. 26, n.2, p. 93-104, 2013.

SILVA, A. J. R. ; ANDRADE, L.H.C. Etnobotânica Nordestina: estudo comparativo da relação entre comunidades e vegetação na Zona do Litoral-Mata do Estado de Pernambuco, Brasil. Acta Botanica Brasilica, v.19, n.1, p.45-60, 2005.

SILVA, V.A.; ANDRADE, L.H.C. Etnobotânica Xucuru: plantas medicinais. Revista Brasileira de Farmácia, v.79, n.1/2, p.33-36, 1998.

SILVA, V.A.; ANDRADE, L.H.C. O significado cultural das espécies botânicas entre indígenas de Pernambuco: o caso Xucuru. Biotemas, v.17, n.1, p.79-94, 2004.

TEIXEIRA, S.A.; MELO, J.I.M. Plantas medicinais utilizadas no município de Jupi, Pernambuco, Brasil. Iheringia, Série Botânica, v.6, n.1/2, p.511, 2006.

VALE, T.G.; FURTADO, E.C.; SANTOS JR, J.G.; VIANA, G.S.B. Central effects of citral, myrcene and limonene, constituents of essential oil chemotypes from Lippia alba (Mill.) N.E. Brown. Phytomedicine, v.9, n.8, p.709-714, 2002.

ZUCCHI, M.R.; OLIVEIRAJUNIOR, V.F.; GUSSONI, M.A.; SILVA, M.B.; SILVA, F.C.; MARQUES, N.E. Levantamento de plantas medicinais na cidade de Ipameri - GO. Revista Brasileira de Plantas Medicinais, v.15, n.2, p.273-279, 2013. 